\title{
A Computationally Efficient Method for Simulating Metal-Nanowire Dipole Antennas at Infrared and Longer Visible Wavelengths
}

\author{
Mario Fernández Pantoja, Member, IEEE, Matthew G. Bray, Member, IEEE, Douglas H. Werner, Fellow, IEEE, \\ Pingjuan L. Werner, Senior Member, IEEE, and Amelia Rubio Bretones, Senior Member, IEEE
}

\begin{abstract}
This paper presents a numerically efficient approach for simulating nanowires at infrared and long optical wavelengths. A computationally efficient circuit-equivalent modeling approach based on the electric-field integral-equation (EFIE) formulation is employed to simulate the highly dispersive behavior of nanowires at short wavelengths. The proposed approach can be used both for frequency-domain and for time-domain EFIE formulations. In comparison with widely used full-wave solutions achieved through the finite-difference time-domain method, the circuit-based EFIE formulation results in a sharp reduction of the computational resources while retaining high accuracy.
\end{abstract}

Index Terms-Computational nanotechnology, integral equations, time-domain analysis.

\section{INTRODUCTION}

T HE development of experimental techniques for manufacturing nanoscale-diameter wires [1] has garnered a lot of recent interest in their use as antennas at optical wavelengths [2]-[6]. The unique performance of these nanoscale structures has also been demonstrated through numerical simulations of metal nanowires [7], [8], which usually involve incorporating experimental measurements of the optical material parameters at a discrete set of wavelengths [9], [10]. Carbon nanotubes [11] have also been explored, based either on circuital circuit models inferred through a quantum approach [12] or on accurate descriptions of the interband and intraband motion of

Manuscript received April 5, 2010; revised December 13, 2010; accepted February 8, 2011. Date of publication February 22, 2011; date of current version March 9, 2012. This work was supported in part by the Spanish Ministry of Education under Project PR2009-0443, in part by the Penn State MRSEC under NSF Grant 0213623, in part by the EU FP7/2007-2013 under Grant GA 205294 (HIRF SE project), in part by the Spanish National Projects TEC2010-20841C04-04, CSD200800068, and DEX-5300002008105, and in part by the Junta de Andalucia Project P09-TIC5327. The review of this paper was arranged by Associate Editor C. Zhou.

M. F. Pantoja and A. R. Bretones are with the Department of Electromagnetismo y Fisica de la Materia, Universidad de Granada, Granada 18071, Spain (e-mail: mario@ugr.es; arubio@ugr.es).

M. G. Bray is with the Lockheed Martin Commercial Space System, Newtown, PA 18940 USA (e-mail: mattgbray@gmail.com).

D. H. Werner and P. L. Werner are with the Department of Electrical Engineering, The Pennsylvania State University, University Park, PA 16802 USA (e-mail: dhw@psu.edu; plw7@psu.edu). electrons in nanostructures [13]. Metal-nanowire antennas have been predicted to outperform carbon-nanotube antennas in the optical range [11] while the opposite is true at far-infrared and terahertz frequencies [14], [15]. Numerical simulations of other optical structures have been performed for cylindrical waveguides [16], metal nanoparticles [17], and nanostructured metal films [18]. Furthermore, optical antennas have been studied using both analytical [19] and experimental [20] approaches and a technique for tuning the performance of optical nanodipoles has even been proposed in [21] and [22] through the insertion of nanorods [23], [24].

Several numerical methods for analyzing metal nanowires have been successfully used in previous papers. Among these methods, full-wave solutions of arrays of silver nanowires were achieved by using finite-difference time-domain (FDTD) [25], [26], and metal nanodipoles were simulated by the finiteelement approach [7]. On the other hand, integral-equation solutions were reached by means of volumetric integral equations applied to silver nanowires [27], or by using surface impedance integral equations (SI-IE) [8] for infinitely long cylinders consisting of silica nanowires [28] and dielectric-coated nanowires [29]. Despite the accuracy of the finite-difference and finiteelement techniques, the considerable computational resources required even for the simulation of relatively simple nanostructures constitute a serious drawback when more complex structures are considered, such as arrays or bundles of nanowires. For this reason, there has been recent interest in developing integralequation-based methods that offer solutions many orders of magnitude faster than FDTD or finite-element techniques. The application of SI-IE, compared with volumetric integral equations, is advantageous in terms of computational resources, its main drawback being that only a handful of nanostructures can be studied under this approach.

In this paper, we propose an efficient scheme to simulate metal nanowires at near-infrared and longer optical regimes by using an SI-IE approach, which applies a thin-wire approximation that avoids the use of volumetric integral equations. Other foundations of this technique are based on Lorentz-Drude phenomenological models that were developed experimentally for the characterization of the optical properties of optoelectronic devices [30]. These characterizations are used to infer equivalent electric models, both in frequency-domain [31], [32] and in time-domain [33] electric-field integral-equation (EFIE) formulations, which allow a fast simulation of different metal nanowires. 


\section{COMPutATional Model of NANOWiRES}

The numerical simulation of nanowires in the optical regime (400-790 THz) requires the accurate incorporation of the appropriate electromagnetic constitutive parameters. In this paper, we use phenomenological models based on a Lorentz-Drudedamped oscillator for describing the optical properties of different metal solids [30]. A computationally efficient method of including these constitutive parameters in an electromagnetic solver is presented through the use of equivalent circuit models. Integral equation solvers that employ these models are very efficient in terms of memory and computational time in comparison with other full-wave solvers such as the FDTD method. It was demonstrated in [8] that nanowires at near infrared and the lower part of the visible spectrum can be accurately simulated using numerical methods based on integral equations.

\section{A. Lorentz-Drude Model for Metals at Optical Wavelengths}

The optical constants of metals can be represented by using a Lorentz-Drude material model that accounts for both intraband (related to the movement of free electrons in the conduction band) and interband (associated with transitions between valence and conduction bands) effects [10]. Thus, the complex dielectric function can be expressed in the form $\varepsilon_{r}(\omega)=\varepsilon_{r}^{\mathrm{ib}}(\omega)+\varepsilon_{r}^{\mathrm{eb}}(\omega)$, where the intraband contribution is described by the free-electron Drude model

$$
\varepsilon_{r}^{\mathrm{ib}}(\omega)=1-\frac{f_{0} \omega_{p}^{2}}{\omega\left(\omega-i \Gamma_{0}\right)}
$$

with $\omega_{p}$ corresponding to the plasma frequency associated with the metal. Intraband transitions are characterized by an oscillator strength $f_{0}$ and a damping constant $\Gamma_{0}$. The term corresponding to the interband contribution obeys a simple semiquantum model expressed in a Lorentz form given by

$$
\varepsilon_{r}^{\mathrm{eb}}(\omega)=\sum_{j=1}^{K} \frac{f_{j} \omega_{p}^{2}}{\left(\omega_{j}^{2}-\omega^{2}\right)+i \omega \Gamma_{j}}
$$

where $K$ represents the number of oscillators needed to achieve a reasonable fit to the experimental data. Each of these oscillators is described by three parameters corresponding to their frequency $\omega_{j}$, strength $f_{j}$, and damping constant $\Gamma_{j}$. Numerical values of these modeling parameters for different metals at optical wavelengths can be found in [30].

\section{B. Circuit-Equivalent Representation of the Lorentz-Drude Model}

The dispersive dielectric permittivity can be represented as a frequency-dependent impedance per unit length. In the case of the Lorentz-Drude model, intraband- and interband-equivalent conductivities ( $\sigma^{\mathrm{ib}}$ and $\sigma^{\mathrm{eb}}$, respectively) can be derived from the generic formula

$$
\sigma(\omega)=i \omega \varepsilon_{0}\left[\varepsilon_{r}(\omega)-1\right] .
$$

Therefore, the equivalent resistivity $\rho(\omega)$ can be specified as impedance per unit length for any metal. If we let $i \omega=s$, then

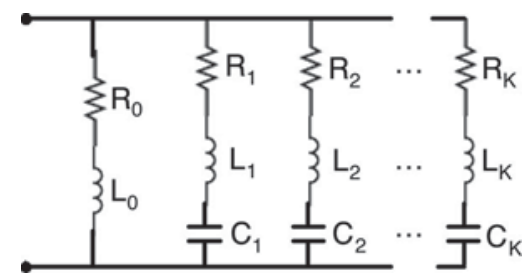

Fig. 1. Circuit equivalent (per unit length) of the Lorentz-Drude model.

TABLE I

RESISTANCES, INDUCTANCES, AND CAPACITANCES (IN $\Omega \cdot \mathrm{m}, \mathrm{H} \cdot \mathrm{m}$, AND F/m, Respectively) OF SOME TyPical Metals

\begin{tabular}{c|c|c|c|c}
\hline & $A g$ & $A l$ & $A u$ & $C u$ \\
\hline $\boldsymbol{R}_{\theta}$ & $5.202 \cdot 10^{-8}$ & $2.977 \cdot 10^{-8}$ & $6.358 \cdot 10^{-8}$ & $3.307 \cdot 10^{-8}$ \\
$\boldsymbol{L}_{\theta}$ & $7.134 \cdot 10^{-22}$ & $4.169 \cdot 10^{-22}$ & $7.896 \cdot 10^{-22}$ & $7.256 \cdot 10^{-22}$ \\
\hline $\boldsymbol{R}_{\boldsymbol{I}}$ & $5.475 \cdot 10^{-5}$ & $4.860 \cdot 10^{-7}$ & $9.155 \cdot 10^{-6}$ & $3.927 \cdot 10^{-6}$ \\
$\boldsymbol{L}_{\boldsymbol{I}}$ & $9.274 \cdot 10^{-21}$ & $9.607 \cdot 10^{-22}$ & $2.500 \cdot 10^{-20}$ & $6.840 \cdot 10^{-21}$ \\
$\boldsymbol{C}_{\boldsymbol{l}}$ & $7.016 \cdot 10^{-11}$ & $1.718 \cdot 10^{-8}$ & $1.006 \cdot 10^{-10}$ & $7.480 \cdot 10^{-10}$ \\
\hline $\boldsymbol{R}_{2}$ & $3.338 \cdot 10^{-6}$ & $2.067 \cdot 10^{-6}$ & $3.145 \cdot 10^{-5}$ & $6.436 \cdot 10^{-6}$ \\
$\boldsymbol{L}_{2}$ & $4.861 \cdot 10^{-21}$ & $4.361 \cdot 10^{-21}$ & $6.001 \cdot 10^{-20}$ & $4.012 \cdot 10^{-21}$ \\
$\boldsymbol{C}_{2}$ & $4.438 \cdot 10^{-12}$ & $4.167 \cdot 10^{-11}$ & $1.048 \cdot 10^{-11}$ & $1.235 \cdot 10^{-11}$ \\
\hline $\boldsymbol{R}_{3}$ & $5.411 \cdot 10^{-6}$ & $2.696 \cdot 10^{-6}$ & $1.117 \cdot 10^{-5}$ & $2.816 \cdot 10^{-6}$ \\
$\boldsymbol{L}_{3}$ & $5.480 \cdot 10^{-20}$ & $1.313 \cdot 10^{-21}$ & $8.453 \cdot 10^{-21}$ & $5.771 \cdot 10^{-22}$ \\
$\boldsymbol{C}_{3}$ & $1.180 \cdot 10^{-13}$ & $1.008 \cdot 10^{-10}$ & $5.815 \cdot 10^{-12}$ & $2.672 \cdot 10^{-11}$ \\
\hline $\boldsymbol{R}_{4}$ & $9.986 \cdot 10^{-7}$ & $3.734 \cdot 10^{-5}$ & $3.783 \cdot 10^{-6}$ & $4.277 \cdot 10^{-6}$ \\
$\boldsymbol{L}_{4}$ & $7.176 \cdot 10^{-22}$ & $7.269 \cdot 10^{-21}$ & $9.986 \cdot 10^{-22}$ & $6.539 \cdot 10^{-22}$ \\
$\boldsymbol{C}_{4}$ & $7.318 \cdot 10^{-12}$ & $4.941 \cdot 10^{-12}$ & $2.342 \cdot 10^{-11}$ & $5.300 \cdot 10^{-12}$ \\
\hline $\boldsymbol{R}_{5}$ & $3.923 \cdot 10^{-7}$ & & $4.604 \cdot 10^{-7}$ & \\
$\boldsymbol{L}_{5}$ & $1.067 \cdot 10^{-22}$ & & $1.369 \cdot 10^{-22}$ & \\
$\boldsymbol{C}_{5}$ & $9.857 \cdot 10^{-12}$ & & $1.783 \cdot 10^{-11}$ & \\
\hline \hline
\end{tabular}

the resistivity $\rho(\omega)$ may be expressed as follows:

$$
\frac{1}{\rho(\omega)}=\sigma^{\mathrm{ib}}(\omega)+\sigma^{\mathrm{eb}}(\omega)=\frac{f_{0} \omega_{p}^{2}}{s+\Gamma_{0}}+\sum_{j=1}^{K} \frac{s \varepsilon_{0} f_{j} \omega_{p}^{2}}{s^{2}+s \Gamma_{j}+\omega_{j}^{2}} .
$$

By defining a set of inductances $L_{j}$, capacitances $C_{j}$, and resistances $R_{j}$, per unit length, such that

$$
\begin{aligned}
L_{j} & =\frac{1}{\varepsilon_{0} f_{j} \omega_{p}^{2}} \\
C_{j} & =\frac{1}{\omega_{j}^{2} L_{j}} \\
R_{j} & =\Gamma_{j} L_{j}
\end{aligned}
$$

then (4) can be cast in the form

$$
\frac{1}{\rho(\omega)}=\frac{1}{R_{0}+s L_{0}}+\sum_{j=1}^{K} \frac{1}{R_{j}+s L_{j}+\frac{1}{s C_{j}}} .
$$

Fig. 1 shows a schematic representation of the input impedance $Z_{\text {in }}(\omega)$ of the circuit model that can be derived from the equivalent resistivity $\rho(\omega)$ of the metal by considering the length of any piece of the wire. Table I shows the corresponding parameters for four different metals that are commonly used to 
manufacture nanowires, derived from the values of $f_{j}, \omega_{j}, \Gamma_{j}$, and $\omega_{p}$ found in [30]. The silver and gold models require one $R L$ circuit to represent the Drude term and four $R L C$ circuits to represent the Lorentzian responses, whereas aluminum and copper require only one $R L$ circuit and three $R L C$ circuits in parallel.

\section{EFIE for the Simulation of Nanowires}

Electromagnetic scattering or radiation problems regarding nanowires can be solved by employing numerical simulations based on the EFIE. Nanowires usually have a radius that is much smaller than their length and can be modeled by the thin-wire approximation where the axial current is assumed to dominate. Also, the thin-wire approach can be further simplified by considering a particular case derived from the exact Greens function referred to as the approximate kernel, which takes an advantage of the cylindrical symmetry of the sources and avoids the singularities that arise in the general case, by treating the total current as a filament on the wire axis and enforcing the boundary condition on the wire surface. The use of this approach proves to be the key factor for achieving high computational efficiency. Taking into account the loads for the nanowire circuit model illustrated in Fig. 1, the modified frequency-domain Pocklington's EFIE in a vacuum is [31], [32]

$$
\begin{aligned}
{\left[\vec{E}^{i}(\vec{r}, \omega)\right]_{\tan }=} & \frac{i}{4 \pi \varepsilon_{0} \omega}\left[\int_{C^{\prime}} \frac{\omega^{2}}{c^{2}} \frac{e^{-j k R}}{R} \vec{I}\left(\vec{r}^{\prime}, \omega\right) d s^{\prime}\right]_{\tan } \\
& -\frac{i}{4 \pi \varepsilon_{0} \omega}\left[\int_{C^{\prime}} I\left(\vec{r}^{\prime}, \omega\right) \nabla \frac{\partial}{\partial s^{\prime}}\left(\frac{e^{-j k R}}{R}\right) d s^{\prime}\right]_{\tan } \\
& +\left[Z_{\text {in }}(\omega) \vec{J}(\vec{r}, \omega)\right]_{\tan }
\end{aligned}
$$

and the corresponding time-domain EFIE is given by [33]

$$
\begin{aligned}
{\left[\vec{E}^{i}(\vec{r}, t)\right]_{\tan }=} & \frac{1}{4 \pi \varepsilon_{0}}\left[\int_{C^{\prime}} \frac{1}{c^{2} R} \frac{\partial}{\partial t} \vec{I}\left(\vec{r}^{\prime}, t^{\prime}\right) d s^{\prime}\right]_{\tan } \\
& +\frac{1}{4 \pi \varepsilon_{0}}\left[\int_{C^{\prime}} \frac{\vec{R}}{R^{3}}\left(\int_{0}^{t^{\prime}} \frac{\partial}{\partial r^{\prime}} I\left(\vec{r}^{\prime}, \tau\right) d \tau\right) d s^{\prime}\right]_{\tan } \\
& +\frac{1}{4 \pi \varepsilon_{0}}\left[\int_{C^{\prime}} \frac{\vec{R}}{c R^{2}} \frac{\partial}{\partial r^{\prime}} I\left(\vec{r}^{\prime}, t^{\prime}\right) d s^{\prime}\right]_{\tan } \\
& +\mathcal{L}^{-1}\left[Z_{\text {in }}(\omega) \vec{J}(\vec{r}, \omega)\right]_{\tan }
\end{aligned}
$$

where $\mathcal{L}^{-1}$ means for denotes the inverse Laplace transform, and the retarded time between the source and field points is represented as $t^{\prime}=t-(R / c) \cdot \vec{I}$ and $\vec{J}$ account for the unknown total current and current density, respectively, along the arclength $C^{\prime}$ of the thin wire embedded in a vacuum, and they are related through the radius of the nanowire $a$ by using $\vec{I}=2 \pi a \vec{J}$. Also, $R$ accounts for the distance between field and source points, $\vec{E}^{i}$ corresponds to the incident electric field on the surface of the nanowire, and the operator [ $]_{\tan }$ denotes the tangential component to the wire axis of the vector field inside the brackets.

At this point, some considerations about regarding the proposed method are due. First of all, it bears remarking that (9) and (10) are no longer valid in the upper part of the visible spectrum where the skin effect begins to become appreciable and the axial current no longer dominates [8]. Regarding the contribution of the circuital circuit model to the efficiency of the method, it is noticeable that any computational improvement in the solution of (9) is achieved by using that model instead of the equivalent resistivity $\rho(\omega)$, apart from the physical insight that can be inferred by an inspection of the resisitive and reactive values corresponding to the specific circuit elements. Nevertheless, the use of the circuital circuit model in (10) avoids the employment of any numerical inverse Laplace transform in the code. Numerical algorithms for the calculation of $\mathcal{L}^{-1}$ should be avoided because results may suffer either from inaccuracies, as a result of a numerical truncation of the time-domain response associated with the first term on the right-hand side of (10), or from extremely poor performance if the complete response of the same term is considered. By using the $R L C$ circuital circuit model as in [33], the contribution at each segment defined in the method-of-moment solution can be taken into account numerically by a trapezoidal integration or a finite difference approximation of the circuital circuit response. This not only avoids the calculation of derivative and integral forms of the electric current but also enables an effective means of simulating large and complex networks of electric circuits. Then, each $i$ th segment of the nanowire can be replaced by a series of a numerically equivalent resistance ${ }^{\mathrm{nw}} R^{e}$ and a numerically equivalent time-varying field ${ }^{\mathrm{nw}} E_{i}^{e, j}$, derived from the values $R_{m}^{e}$ and $E_{i, m}^{e, j}$ corresponding to the $m$ th branch of the circuital circuit model:

$$
\begin{aligned}
R_{m}^{e} & =R_{m}+\frac{2 L_{m}}{\Delta t}+\frac{\Delta t}{2 C_{m}} \\
E_{i, m}^{e, j} & =E_{i, m}^{C, j}-E_{i, m}^{L, j}+\left(\frac{\Delta t}{2 C_{m}}-\frac{2 L_{m}}{\Delta t}\right) I_{i, m}^{j}
\end{aligned}
$$

where $j$ is the index of a particular time $j \Delta t$, and $I_{i, m}^{j}$ is the current at $m$ th branch of the $i$ th segment. $E_{i, m}^{e, j}$ is the reactive energy stored at that branch, and it is calculated by using

$$
\begin{aligned}
& E_{i, m}^{L, j}=\frac{2 L_{m}}{\Delta t} I_{i, m}^{j}-\left(E_{i, m}^{L, j-1}+\frac{2 L_{m}}{\Delta t} I_{i, m}^{j-1}\right) \\
& E_{i, m}^{C, j}=\frac{\Delta t}{2 C_{m}} I_{i, m}^{j}+\left(E_{i, m}^{C, j-1}+\frac{\Delta t}{2 C_{m}} I_{i, m}^{j-1}\right) .
\end{aligned}
$$

Therefore, the aforementioned equivalent parameters ${ }^{\mathrm{nw}} R^{e}$ and ${ }^{\mathrm{nw}} E_{i}^{e, j}$ at each $i$ th segment are calculated by

$$
\begin{aligned}
{ }^{\mathrm{nw}} R^{e} & =\left(\sum_{m=0}^{K} \frac{1}{R_{m}^{e}}\right)^{-1} \\
{ }^{\mathrm{nw}} E_{i}^{e, j} & ={ }^{\mathrm{nw}} R^{e} \sum_{m=0}^{K} \frac{E_{i, m}^{e, j}}{R_{m}^{e}}
\end{aligned}
$$

resulting in an operational expression of (10) given by

$$
\begin{aligned}
\hat{s} \cdot \vec{E}^{i}(\vec{r}, j \Delta t)= & \frac{1}{4 \pi \varepsilon} \int_{C^{\prime}} \frac{\hat{s} \cdot \hat{s}^{\prime}}{c^{2} R} \frac{\partial}{\partial t} I\left(s^{\prime}, t^{\prime}\right) d s^{\prime} \\
& +\frac{1}{4 \pi \varepsilon} \int_{C^{\prime}} \frac{\hat{s} \cdot \vec{R}}{R^{3}}\left(\int_{0}^{t^{\prime}} \frac{\partial}{\partial r^{\prime}} I\left(s^{\prime}, \tau\right) d \tau\right) d s^{\prime}
\end{aligned}
$$




$$
\begin{aligned}
& +\frac{1}{4 \pi \varepsilon} \int_{C^{\prime}} \frac{\hat{s} \cdot \vec{R}}{c R^{2}} \frac{\partial}{\partial r^{\prime}} I\left(\vec{r}^{\prime}, t^{\prime}\right) d s^{\prime} \\
& +\sum_{i=1}^{N_{s}} \frac{1}{\Delta s}\left\{I_{i}(s, j \Delta t)^{\mathrm{nw}} R^{e}+{ }^{\mathrm{nw}} E_{i}^{e, j}\right\}
\end{aligned}
$$

where $\hat{s}$ is the tangential unit vector to the axis of the wire, and $s^{\prime}$ is the local coordinate along the axis. It should be noted that in order to achieve satisfactory results at the feeding point, the delta-gap model is applied by assuming an additional voltage source placed in parallel with the equivalent circuit model. It is important to note that (17) does not include any numerical inverse Laplace transform, and thus provides higher computational efficiency.

\section{RESULTS AND DISCUSSION}

Solutions to (9) and (17) can be derived by applying the method of moments [32]. On one hand, the nanowires are modeled as straight wire segments using a sinusoidal basis function for the spatial dependence of the electric current in (9). On the other hand, 2-D Lagrangian basis functions are used for both the spatial and temporal dependence of the current in (17). The resulting matrix equations are obtained by applying the pointmatching technique that uses delta functions to project onto the surface of the nanowire in the spatial and temporal dimensions for the time-domain EFIE [31], [33].

Therefore, the formulations of (9) and (17) were verified by comparing the results with simulations performed via the FDTD method [26]. Figs. 2 and 3 show a comparison of the input impedance for nanowire dipoles with lengths of 220 and $440 \mathrm{~nm}$, respectively, simulated using the FDTD method, the frequencydomain integral-equation (FDIE) method, and the time-domain integral-equation (TDIE) method. According to the numerical requirements of each method for frequencies up to $800 \mathrm{THz}$, spatial domains were modeled with segments of $10 \mathrm{~nm}$ for the integral equations, and with a Yee cell size of $2 \mathrm{~nm}$ for the FDTD simulation. Boundary conditions were included in the FDTD by using a perfectly matched layer to terminate the computational grid. The simulation was run for 400000 time steps that took approximately $35 \mathrm{~h}$ on $2.4-\mathrm{GHz}$ Intel Xeon. Simulations of both integral-equation-based methods in the time domain and frequency domain were made using a spatial length-to-radius of 2 per segment, with a total computational time of roughly $20 \mathrm{~s}$, including all frequencies. In both cases, the nanowires had a radius of $10 \mathrm{~nm}$ and were fed in the center using a voltage source modeled with the delta-gap approach. The results from FDTD and TDIE have been transformed to the frequency domain in a postprocessing step for comparison with the FDIE method.

Reasonable agreement was achieved between the three different methods, but differences are noticed between the 220 and $440 \mathrm{~nm}$ dipoles in the upper part of the visible region (from about 500-600 THz). In the optical regime, the surface-impedance approximation assumes that the main mode is dominant over the branch-cut contributions, albeit this is true only for points on the antenna located far enough from the source. For small radius values, which present high attenuation as the current propagates

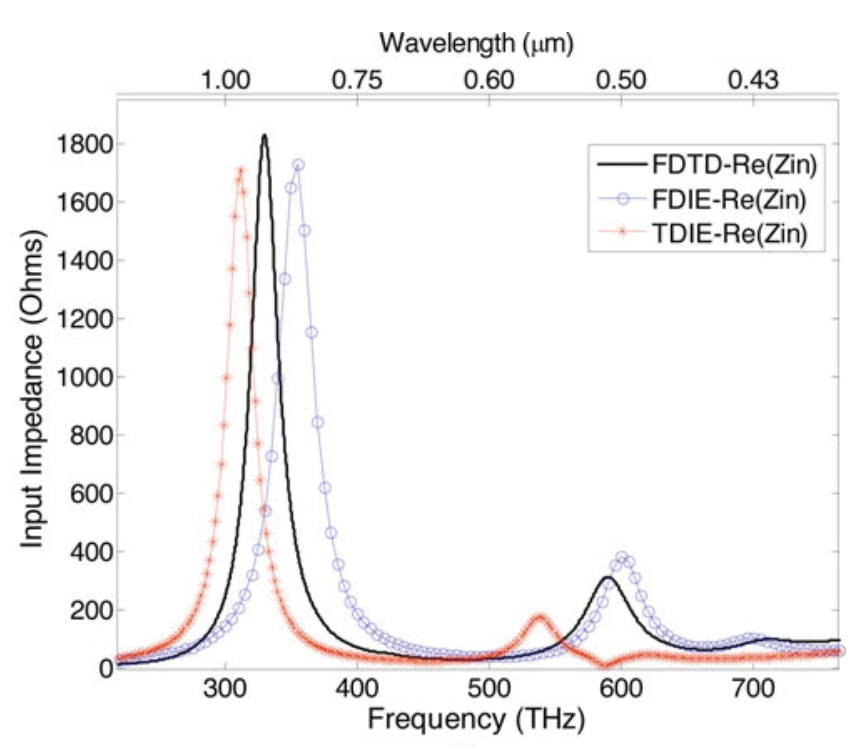

(a)

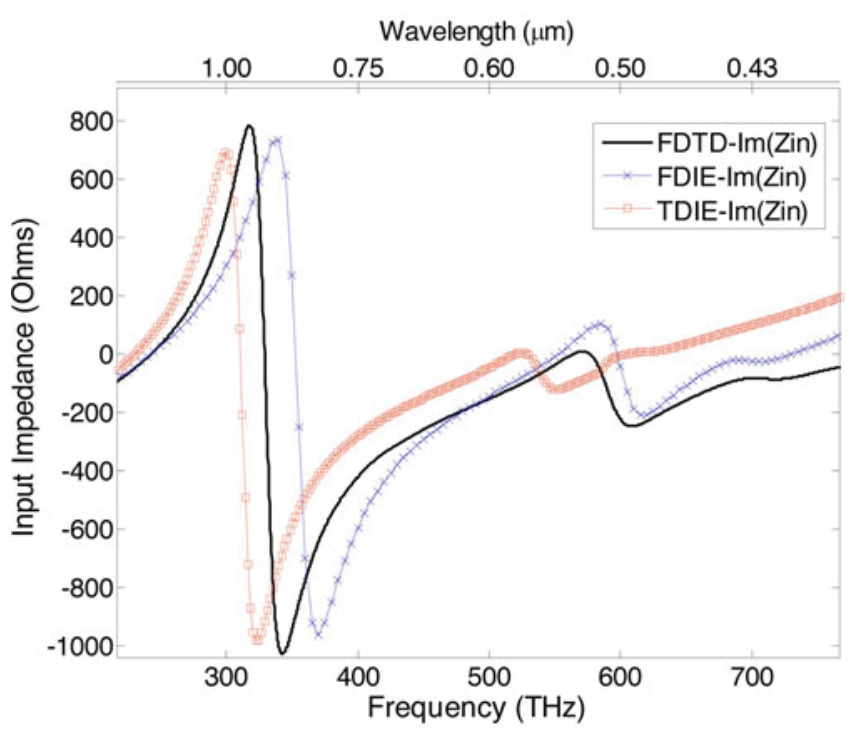

(b)

Fig. 2. (a) Real and (b) imaginary parts of the input impedance of nanowire dipole of length $220 \mathrm{~nm}$ and radius $10 \mathrm{~nm}$, fed at its center and modeled using different numerical methods.

along the dipole, the former condition holds somewhat. However, the combined degradation of the two main assumptions of the method for large-radius dipoles - the thin-wire approximation and the domination of the main branch in the surface impedance approach—leads to serious inaccuracies especially in the upper part of the optical range.

The differences in the infrared region seen in Fig. 2 are mainly due to the low ratio between the length and radius of the dipole, which limits the number of segments that can be used to model the nanoantennas for the integral-equation-based solutions. This leads to a degradation of the thin-wire approximation. Taking the FDTD solution for reference, percentages of error in the impedance values determined with FDIE and TDIE have been calculated. For the peak values at the first resonance, these errors are below 5\% in the case presented in Fig. 2. Nevertheless, 


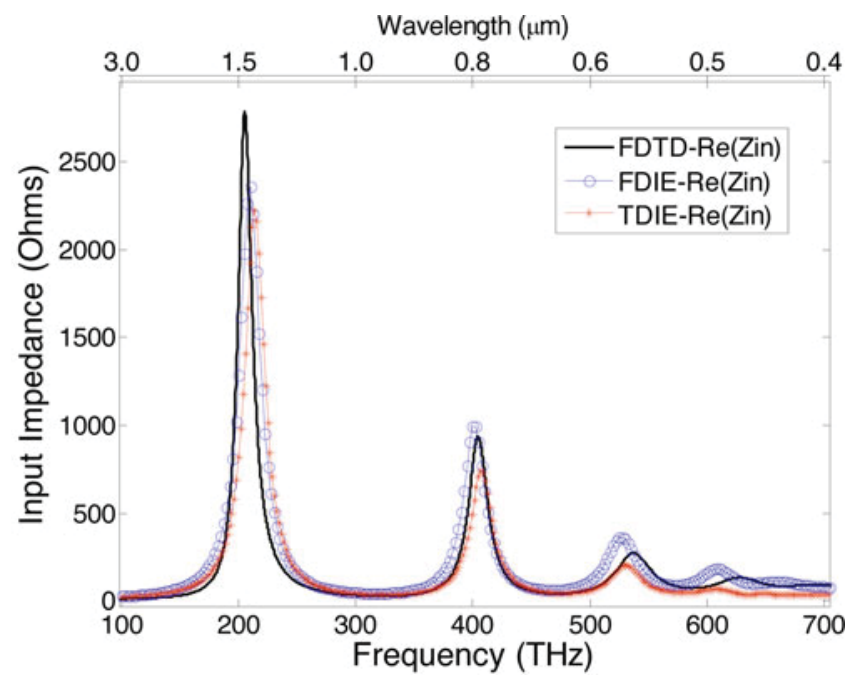

(a)

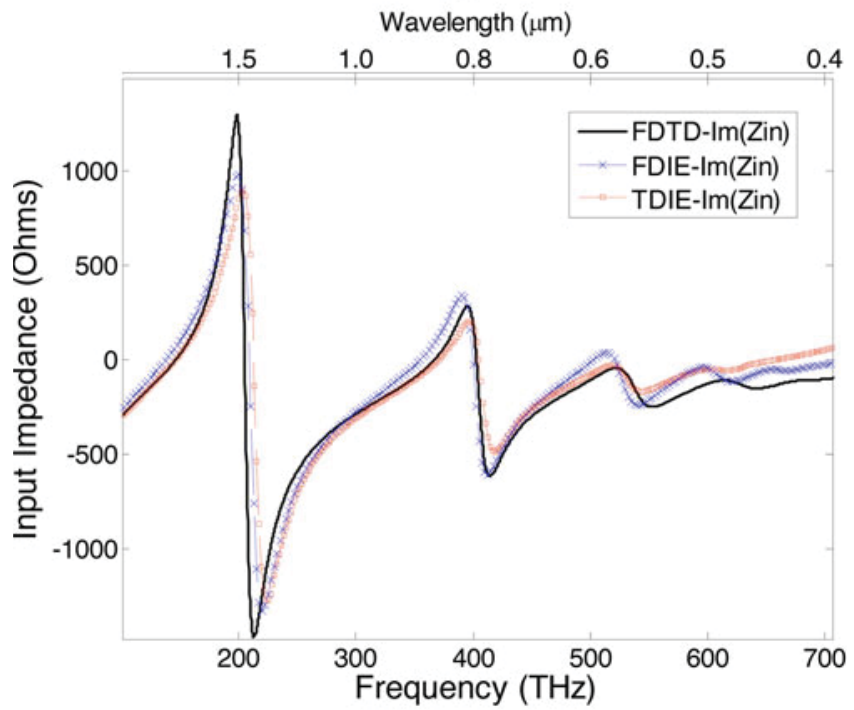

(b)

Fig. 3. (a) Real and (b) imaginary parts of the input impedance of nanowire dipole of length $440 \mathrm{~nm}$ and radius $10 \mathrm{~nm}$, fed at its center and modeled using different numerical methods.

there is a shift in the resonance frequency that can be considered tolerable for the FDIE solution (3.1\% percentage error) but this is unacceptable for the TDIE solution (12.5\% error and a wavelength change of $150 \mathrm{~nm}$ ). Therefore, it can be seen that for cases where the thin-wire approximation is no longer valid the proposed approach is suitable only as a rough estimation, and the use of other assessments based on analytical methods should be considered [16], [19]. Further improvements of this technique could arise by combining thin-wire integral-equation solutions based on the exact kernel, which have been shown to be accurate for low length-to-radius ratios [34], and considering higher order propagation modes predicted in nanowires at infrared and optical regimes [8]. However, the drastic reduction in computational time through the use of integral equations greatly compensates for the reduction in accuracy, always bearing in mind that integral equations provide only a preliminary estimate, which, although coarse, can be still quite useful for

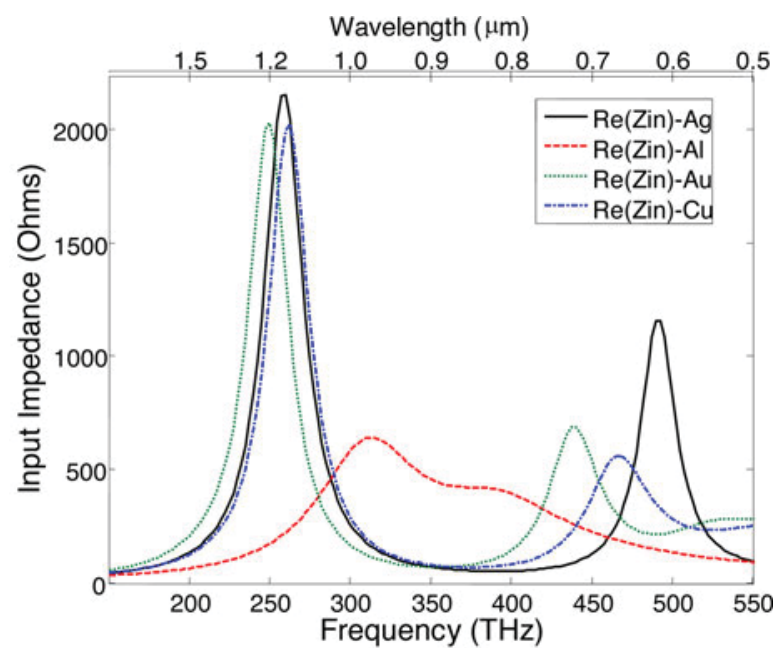

(a)

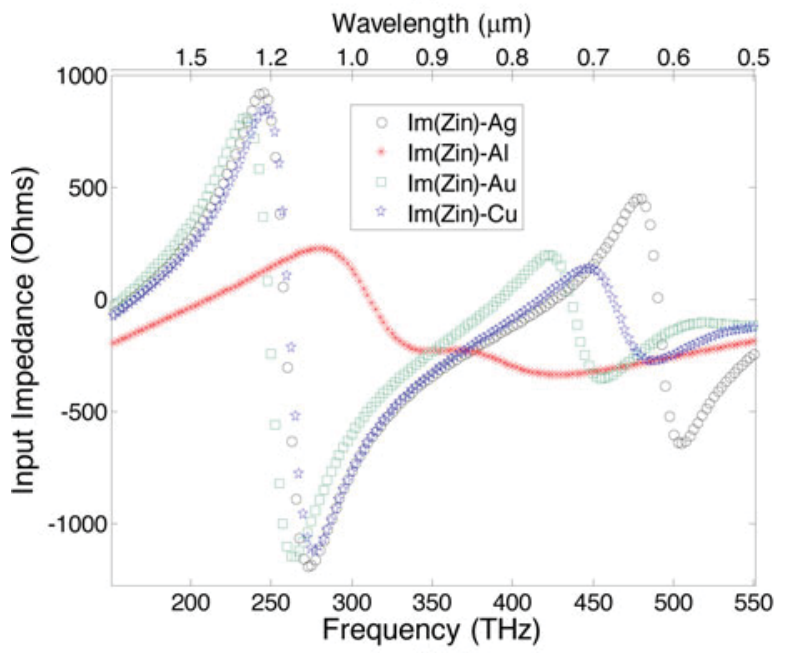

(b)

Fig. 4. (a) Real and (b) imaginary parts of the input impedance of several nanowire dipoles of length $440 \mathrm{~nm}$ and radius $10 \mathrm{~nm}$.

the initial evaluation of antenna characteristics or for the comparison of the behavior of different antennas. On the other hand, looking at Fig. 3, we find that the shift in the resonance frequencies predicted by the different methods is much lower, leading to tolerable errors $(1.9 \%$ and $3.8 \%$ for FDIE and TDIE, respectively) for cases presenting a higher length-to-radius ratio.

Another important question arises from the deviation noted in Figs. 2 and 3 compared to the resonance frequency corresponding to a perfect electric conductor dipole of the same dimensions, i.e., geometrical resonance frequencies. As pointed out in [20] and [23], the intraband and interband transitions of the composing material influence the velocity of the surfacewave propagation mode in the nanodipole, and constitutes a major factor in applied designs. Thus, the main effect of the transitions in different metal-nanowires is a shift of the geometrical resonance frequencies. To confirm this conclusion, we use the aforementioned integral-equation methods to evaluate nanowires having different material properties. Fig. 4 shows the input impedance for gold, silver, aluminum, and copper nanowires, each with a length of $440 \mathrm{~nm}$, a radius of $10 \mathrm{~nm}$, and 


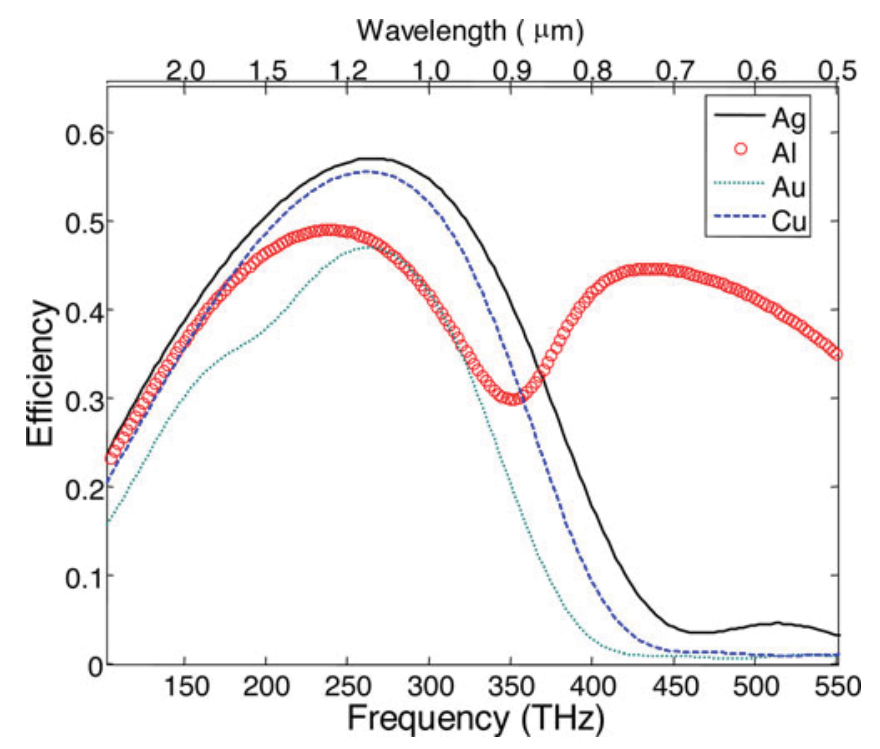

Fig. 5. Efficiency of several metal nanowires dipoles of length $440 \mathrm{~nm}$ and radius $10 \mathrm{~nm}$.

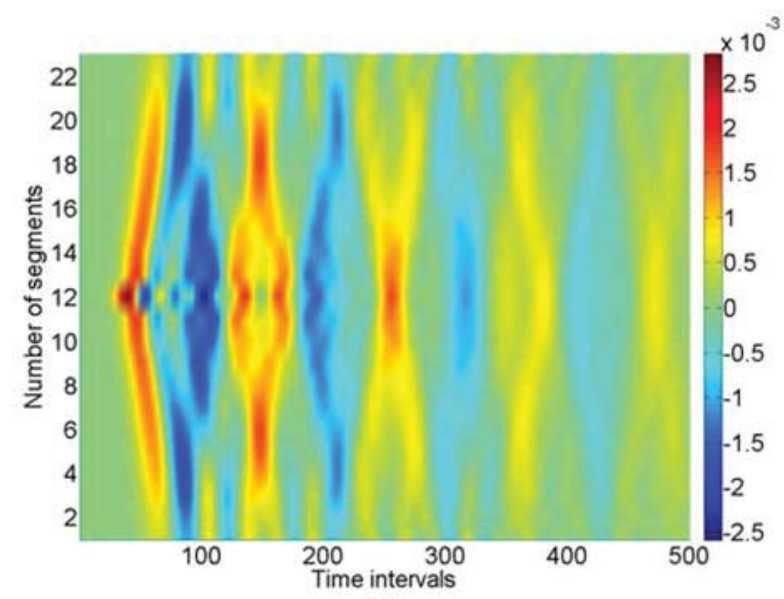

(a)

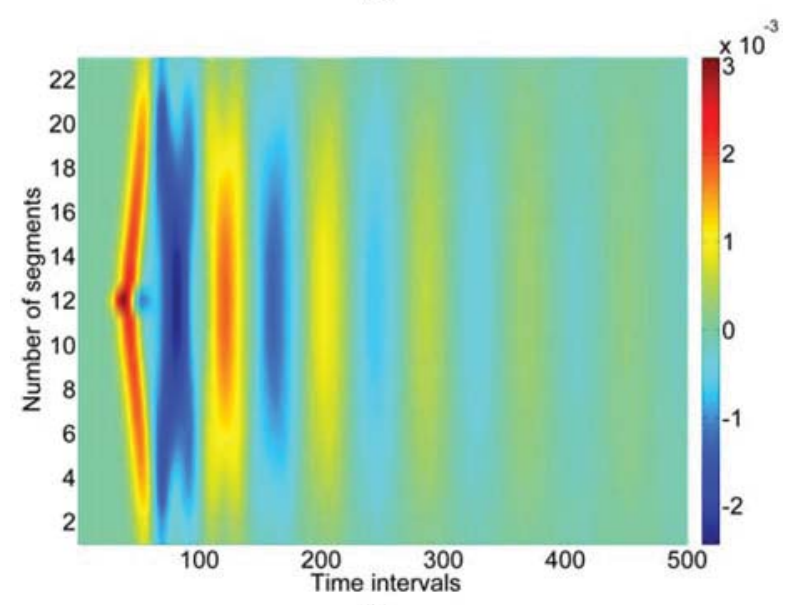

(b)

Fig. 6. Time-domain plot of currents in nanowires of length $440 \mathrm{~nm}$ and radii $10 \mathrm{~nm}$, composed of (a) $\mathrm{Ag}$ and (b) Al. Taking into account that the nanowire has been simulated with a total of 23 segments, each segment of the nanowire ( $Y$-axis) has a length of $19.13 \mathrm{~nm}$, and the time interval chosen in the numerical model ( $X$-axis) corresponds to a duration of $637.68 \mathrm{fs}$.

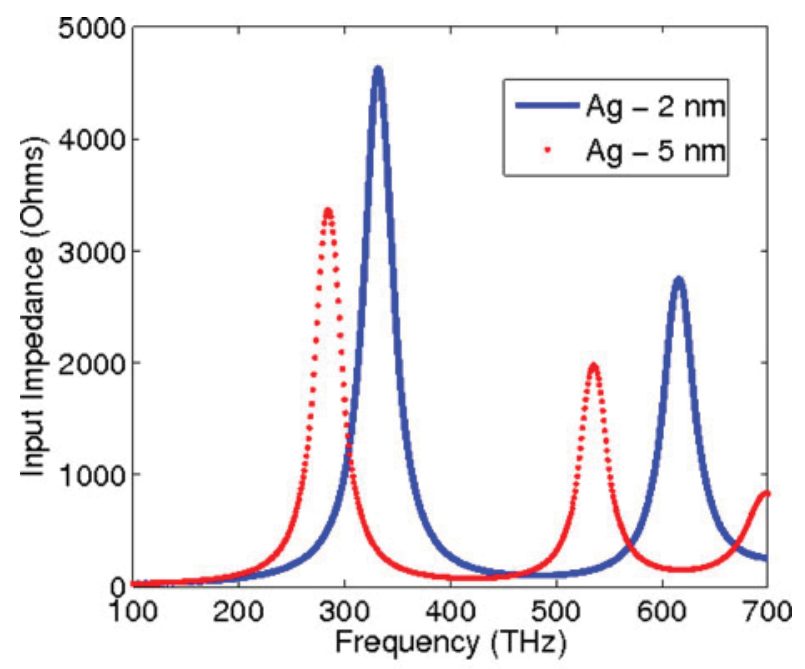

(a)

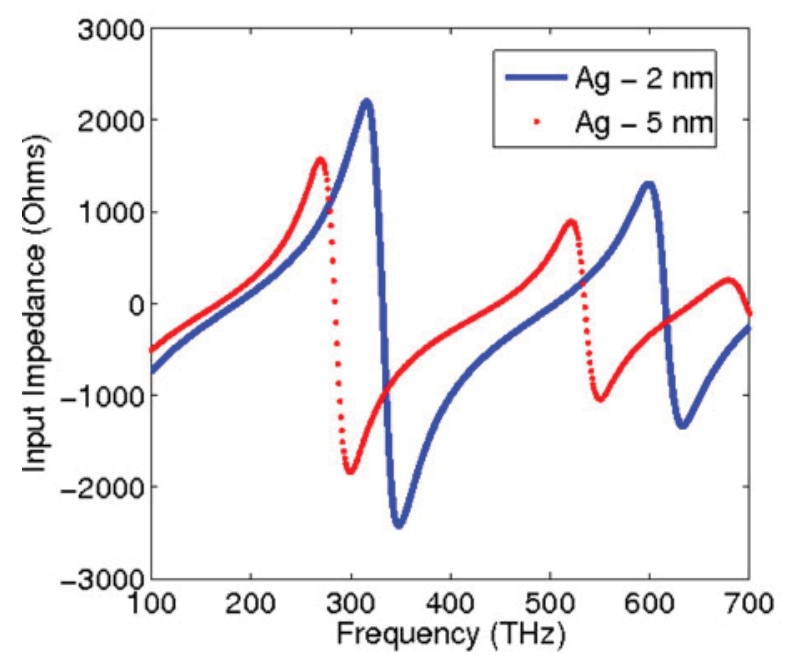

(b)

Fig. 7. (a) Real and (b) imaginary parts of the input impedance for a 440-nm long Ag dipole with different radii.

a feed point located at the center of the wire. Moreover, it is worth noting that the impedance and efficiency of the silver, gold, and copper nanowires are very similar, because all of these metals share the same group in the periodic table, indicating a similar electronic configuration for the external energy levels. Most of the electrical properties (e.g., conductivity) are closely related to the interaction between electrons located at these external energy levels, which leads to their similar frequency response. Furthermore, Fig. 5 shows the corresponding efficiency of these radiators. It can be seen that aluminum has much higher efficiency in the lower part of the visible spectrum. The relatively flat response of the input impedance and efficiency suggests that it is a good option for the design of broadband systems based on nanowires. Moreover, the low resistance and inductance in the interband response of the Lorentz-Drude model (corresponding to $R_{0}$ and $L_{0}$ in Table I) suggest the formation of slow waves for these dipoles. The existence of these slow waves can be confirmed by a comparison of the time-domain currents in the nanowires. Fig. 6 shows a comparison between the current 
on $\mathrm{Ag}$ and $\mathrm{Al}$ nanowire dipoles. It can be seen that the initial current pulse is fed at the center (segment 12) of both the Ag (on the left top ) and the $\mathrm{Al}$ (on the right bottom) nanowires at the same time (at approximately the 30th time interval). After propagating along the wire, the current pulse reaches the end of the dipoles at the 60th time interval for the Ag nanowire, and at the 52nd time interval for the Al nanowire. Therefore, the surface waves on each dipole are seen to have different velocities of propagation as expected.

Finally, the dependence of the results is also demonstrated by varying the radius of the nanowire. It is well known that the influence of this parameter over the performance of the nanodipole is higher than in classical dipoles, because of the change in the velocity of the propagation of the surface wave caused by a variation in the radius. This effect can be inferred by examining the terms in (9) and (10), which contain $Z_{\text {in }}(\omega) \vec{J}(\vec{r}, \omega)$. Thus, as shown in Fig. 7 for a silver nanodipole, a relatively small decrease in the radius affects in a significant way the resonance frequencies.

\section{CONCLUSION}

This paper demonstrates that nanowire simulations based on the EFIE constitute a fast alternative to other full-wave numerical-analysis methods. Moreover, we have shown that physical models of nanowires can be effectively incorporated into the EFIE with only a minor increase in computational resources through the use of circuit-equivalent models for the Lorentz-Drude conductivity. This scheme facilitates the comparison between the resonance frequencies of nanowires simulated with different metals, demonstrating the main influence of the surface-wave velocity in the half-wave resonances. Evaluation of the computational resources needed for EFIE solutions shows a dramatic improvement in efficiency compared to FDTD (20 s versus $35 \mathrm{~h}$ for the examples presented here). Thus, the proposed method is expected to provide an efficient means for the future simulation of nanowire arrays and more complex nanowire structures.

\section{REFERENCES}

[1] C. Xiang, M. A. Thompson, F. Yang, E. J. Menke, L. C. Yang, and R. M. Penner, "Lithographically patterned nanowire electrodeposition," Phys. Stat. Sol., vol. 5, pp. 3503-3505, 2008.

[2] J. Alda, J. M. Rico-García, J. M. López-Alonso, and G. Boreman, “Optical antennas for nano-photonic applications," Nanotechnology, vol. 16, pp. 230-234, 2005.

[3] R. D. Grober, R. J. Schoelkopf, and D. E. Prober, "Optical antenna: Towards a unity efficiency near-field optical probe," Appl. Phys. Lett., vol. 70, no. 11, pp. 1354-1356, 1997.

[4] K. B. Crozier, A. Sundaramurthy, G. S. Kino, and C. F. Quate, "Optical antennas: Resonators for local field enhancement," J. Appl. Phys., vol. 94, p. 4632, 2003.

[5] P. Bharadwaj, B. Deutsch, and L. Novotny, "Optical antennas," Adv. Opt. Photon., vol. 1, pp. 438-483, 2009.

[6] Q.-H. Park, "Optical antennas and plasmonics," Contemporary Phys., vol. 50, no. 2, pp. 407-423, Mar. 2009.

[7] K. Sendur and E. Baran, "Near-field optical power transmission of dipole nano-antennas," Appl. Phys. B, vol. 96, pp. 325-335, 2009.

[8] G. W. Hanson, "On the applicability of the surface impedance integral equation for optical and near infrared copper dipole antennas," IEEE Trans. Antennas Prop., vol. 54, no. 12, pp. 3677-3685, Dec. 2006.
[9] P. B. Johnson and R. W. Christy, "Optical constants of the noble metals," Phys. Rev. B, vol. 6, pp. 4370-4379, 1972.

[10] M. Fox, Optical Properties of Solids, 2nd ed. London, U.K.: Oxford Univ. Press, 2010.

[11] J. Hao and G. W. Hanson, "Infrared and optical properties of carbon nanotube dipole antennas," IEEE.Trans. Nanotech., vol. 5, no. 6, pp. 766775, Nov. 2006.

[12] C. Rutherglen and P. Burke, "Nanoelectromagnetics: Circuit and electromagnetic properties of carbon nanotubes," Small, vol. 5, no. 8, pp. 884 906, 2009.

[13] S. A. Maksimenko and G. Y. Slepyan, "Nanoelectromagnetics of lowdimensional structures," in Nanometer Structures: Theory, Modeling and Simulation, A. Lakhtakia, Ed. Bellingham, WA: SPIE, 2004.

[14] G. W. Hanson, "Radiation efficiency of nano-radius antennas in the microwave and far-infrared regimes," IEEE. Antennas Prop. Mag., vol. 50, no. 3, pp. 66-77, Jun. 2008.

[15] M. F. Pantoja, D. H. Werner, P. L. Werner, and A. R. Bretones, "TDIE modeling of carbon nanotube dipoles at microwave and Terahertz bands," IEEE Antennas Wireless Prop. Lett., vol. 9, pp. 32-35, 2010.

[16] L. Novotny and C. Hafner, "Light propagation in a cylindrical waveguide with a complex, metallic dielectric function," Phys. Rev. E., vol. 50, pp. 4094-4106, Nov. 1994.

[17] K. L. Kelly, E. Coronado, L. L. Zhao, and G. C. Schatz, "The optical properties of metal nanoparticles: The influence of size, shape, and dielectric environment," J. Phys. Chem. B, vol. 107, no. 3, pp. 668-677, 2003.

[18] E. X. Jin and X. Xu, "Finite-difference time-domain studies on optical transmission through planar nano-apertures in a metal film," Jpn. J. Appl. Phys., vol. 43, pp. 407-417, 2004.

[19] L. Novotny, "Effective wavelength scaling for optical antennas," Phys. Rev. Lett., vol. 98, article id. 266802, pp. 266802-1-266802-4, 2007.

[20] F. Neubrech, T. Kolb, R. Lovrincic, G. Fahsold, A. Puccia, J. Aizpurua, T. W. Cornelius, M. E. Toimil-Molares, R. Neumann, and S. Karim, "Resonances of individual metal nanowires in the infrared," App. Phys. Lett., vol. 89, p. 253104, 2006.

[21] A. Alu and N. Engheta, "Tuning the scattering response of optical nanoantennas with nanocircuit loads," Nature Photonics, vol. 2, pp. 307-310, May 2008.

[22] A. Alu and N. Engheta, "Input impedance, nanocircuit loading, and radiation tuning of optical nanoantennas," Phys. Rev. Lett., vol. 101, p. 043901, 2008.

[23] G. W. Bryant, F. J. Garcia de Abajo, and J. Aizpurua, "Mapping the plasmon resonances of metallic nanoantennas," Nano Letters, vol. 8, no. 2, pp. 631-636, 2008.

[24] N. Engheta, A. Salandrino, and A. Alu, "Circuit elements at optical frequencies: Nanoinductors, nanocapacitors, and nanoresistors," Phys. Rev. Lett., vol. 95, p. 095504, 2005.

[25] S. K. Gray and T. Kupka, "Propagation of light in metallic nanowire arrays: Finite-difference time-domain studies of silver cylinders," Phys. Rev. B, vol. 68, pp. 405-415, 2003.

[26] G. Chen, J. Wu, Q. Lu, R. H. Gutierrez, Q. Xiong, M. E. Pellen, J. S. Petko, D. H. Werner, and P. C. Eklund, "Optical antenna effect in semiconducting nanowires," Nano Letters, vol. 18, no. 5, pp. 1341-1346, Apr. 2008.

[27] K. Halterman, J. M. Elson, and S. Singh, "Plasmonic resonances and electromagnetic forces between coupled silver nanowires," Phys. Rev. B, vol. 72, p. 075429, 2005.

[28] J. Lou, L. Tong, and Z. Ye, "Modeling of silica nanowires for optical sensing," Opt. Exp., vol. 13, pp. 2135-2140, 2005.

[29] J. Lou, L. Tong, and Z. Ye, "Dispersion shifts in optical nanowires with thin dielectric coatings," Opt. Exp., vol. 14, pp. 6993-6998, 2006.

[30] A. D. Rakic, A. B. Djurisic, J. M. Elazar, and M. L. Majewski, "Optical properties of metallic-films for vertical-cavity optoelectronic devices," App. Opt., vol. 37, pp. 5271-5283, 1998.

[31] G. J. Burke and A. J. Poggio, "Numerical electromagnetic code (NEC) Method of moments," Naval Ocean Systems Center, San Diego, CA, Rep. UCRL-MA-109338, 1980.

[32] R. F. Harrington, Field Computation by Moment Methods. Piscataway, NJ: IEEE Press, 1993.

[33] M. Fernández Pantoja, A. Rubio Bretones, and R. Gómez Martín, "Time domain analysis of thin-wire loaded antennas using integral equations," IEE Proc. Microw. Antennas Propag.., vol. 147, pp. 203-206, 2000.

[34] D. H. Werner, "A method of moments approach for the efficient and accurate modeling of moderately thick cylindrical wire antennas," IEEE Trans. Antennas Propag., vol. 46, no. 3, pp. 373-381, Mar. 1998. 


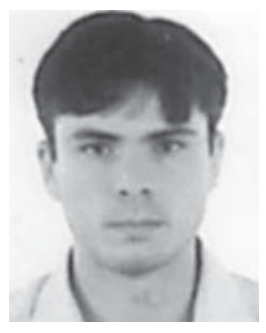

Mario Fernández Pantoja (M’00) received the B.Sc., M.Sc., and Ph.D. degrees in electrical engineering from the University of Granada, Granada, Spain, in 1996, 1998, and 2001, respectively.

From 1997 to 2001, he was an Assistant Professor at the University of Jaen, Jaen, Spain. In 2001, he joined the University of Granada where, in 2004, he became an Associate Professor. He has been a Guest Researcher at the Dipartimento Ingenieria dell'Informazione, University of Pisa, Pisa, Italy, the Antenna and Electromagnetics Group, Denmark Technical University, Lyngby, Denmark, and Computational Electromagnetics and Antenna Research Laboratory, Pennsylvania State University, University Park. His research interests include interaction of electromagnetic waves with structures in time domain and optimization methods applied to antenna design.

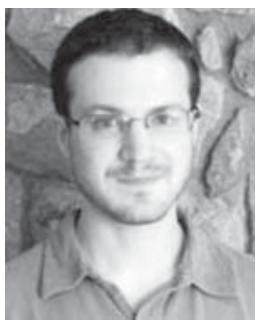

Matthew G. Bray ( S'96-M'05) received the B.E.E. (Hons.) degree in electrical engineering from the University of Delaware, Newark, in 2000, and the M.Sc. and Doctorate of Philosophy degrees from the Pennsylvania State University, University Park, in 2001 and 2005 , respectively.

He is currently with Lockheed Martin Commercial Space System, Newtown, PA. His research interests include metamaterials, bianisotropic media, nanowires, memristors, and the finite difference time domain method. He is also interested in fast optimization techniques and efficient modeling methods.

Dr. Bray is a member of the Tau Beta Pi Engineering Honor Society, the IEEE Antennas and Propagation Society, and the IEEE Microwave Theory and Techniques Society. He has recently served as the Chair and Vice Chair of the Central Pennsylvania Section of the IEEE.

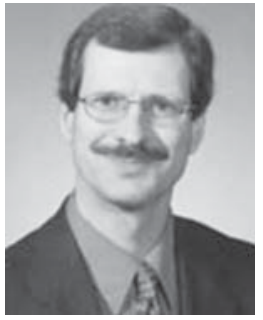

Douglas H. Werner (M'76-SM'81-F'87) received the B.S., M.S., and Ph.D. degrees in electrical engineering and the M.A. degree in mathematics all from The Pennsylvania State University (Penn State), University Park, in 1983, 1985, 1989, and 1986, respectively.

$\mathrm{He}$ is currently a Professor in the Department of Electrical Engineering, Penn State. He is the Director of the Computational Electromagnetics and Antennas Research Lab (CEARL) as well as a member of the Communications and Space Sciences Lab (CSSL) $\mathrm{He}$ is also a Senior Scientist in the Computational Electromagnetics Department of the Applied Research Laboratory and a Faculty Member of the Materials Research Institute (MRI), Penn State. He edited a book entitled Frontiers in Electromagnetics (Piscataway, NJ: IEEE Press, 2000). He has also contributed a chapter for a book entitled Electromagnetic Optimization by Genetic Algorithms (New York: Wiley-Interscience, 1999) as well as for the book entitled Soft Computing in Communications (New York: Springer, 2004). He has recently completed work on a new book (coauthored with R. Haupt) entitled Genetic Algorithms in Electromagnetics (Hoboken, NJ: Wiley/IEEE, 2007). He has also contributed an invited chapter on "Fractal Antennas" for the new edition of the popular Antenna Engineering Handbook (New York: McGrawHill, 2007) as well as a chapter on "Ultra-Wideband Antenna Arrays" for a book entitled Frontiers in Antennas (New York: McGraw-Hill, 2011). He has published more than 450 technical papers and proceedings articles and is the author of eight book chapters. His research interests include theoretical and computational electromagnetics with applications to antenna theory and design, phased arrays, microwave devices, wireless and personal communication systems, wearable and e-textile antennas, conformal antennas, frequency selective surfaces, electromagnetic wave interactions with complex media, metamaterials, electromagnetic bandgap materials, zero and negative index materials, fractal and knot electrodynamics, tiling theory, neural networks, genetic algorithms, and particle swarm optimization.
Dr. Werner was presented with the 1993 Applied Computational Electromagnetics Society (ACES) Best Paper Award and was also the recipient of a 1993 International Union of Radio Science (URSI) Young Scientist Award. In 1994, he received the Pennsylvania State University Applied Research Laboratory Outstanding Publication Award. He is a coauthor (with one of his graduate students) of a paper published in the IEEE TRANSACTIONS ON ANTENNAS AND PROPAGATION, which received the 2006 R.W. P. King Award. He has also received several Letters of Commendation from the Pennsylvania State University Department of Electrical Engineering for outstanding teaching and research. He is a former Associate Editor of Radio Science and an Editor of the IEEE ANTENNAS AND Propagation Magazine. He is a member of the American Geophysical Union (AGU), URSI Commissions B and G, the Applied Computational Electromagnetics Society (ACES), Eta Kappa Nu, Tau Beta Pi, and Sigma Xi. He was the recipient of the College of Engineering PSES Outstanding Research Award and Outstanding Teaching Award in March 2000 and March 2002, respectively. He was also presented with an IEEE Central Pennsylvania Section Millennium Medal. In March 2009, he received the PSES Premier Research Award. He is a Fellow of the IET (formerly IEE) and the ACES.

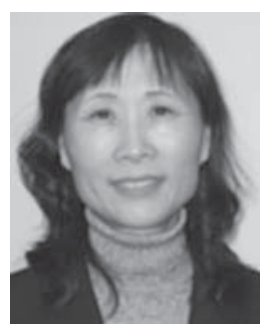

Pingjuan L. Werner (SM'99) received the Ph.D. degree in electrical engineering in 1991 from the Pennsylvania State University, USA.

She is a Professor with the Pennsylvania State University College of Engineering. Her primary research focuses in the area of electromagnetics, including fractal antenna engineering and the application of genetic algorithms in electromagnetics.

Dr. Werner is a member of Tau Beta Pi National Egineering Honor Society, Eta Kappa Nu National Electrical Engineering Honor Society, and Sigma Xi National Research Honor Society.

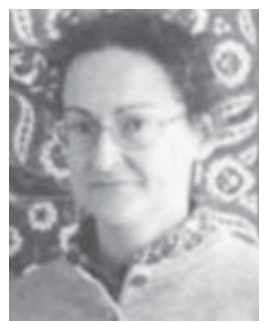

Amelia Rubio Bretones (SM'08) was born in Granada, Spain. She received the Ph.D. (cum laude) degree in physics from the University of Granada, Granada, Spain, in 1988

Since 1985, she has been with the Department of Electromagnetism, University of Granada, first as an Assistant Professor, and then in 1989 as an Associate Professor, and finally, since 2000 as a Full Professor. On several occasions, she was a Visiting Scientist at the Delft University of Technology, Eindhoven University of Technology, and at The Pennsylvania State University. Her research interest is mainly in the field of numerical techniques for applied electromagnetics with an emphasis on time-domain techniques such as finite difference time domain, the application of the method of moments in the time domain for antenna and scattering problems, and hybrid techniques. 